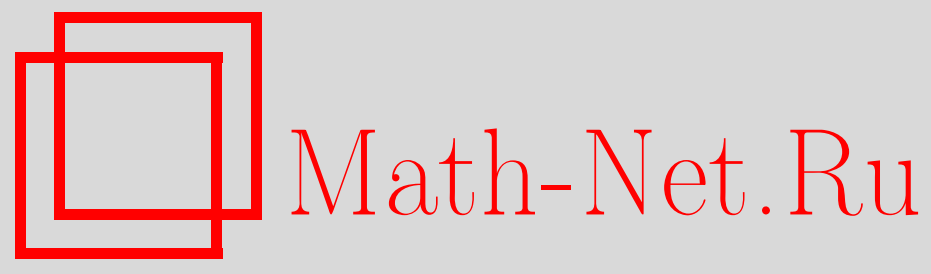

Ю. И. Любич, О. А. Шаталова, Почти евклидовы плоскости в $\ell_{p}^{n}$, Функи. анализ и его прил., 1998, том 32, выпуск 1, 76-78

DOI: https://doi.org/10.4213/faa403

Использование Общероссийского математического портала Math$\mathrm{Net.Ru}$ подразумевает, что вы прочитали и согласны с пользовательским соглашением

http://www . mathnet.ru/rus/agreement

Параметры загрузки:

IP : 52.23 .180 .231

26 апреля 2023 г., 12:39:48

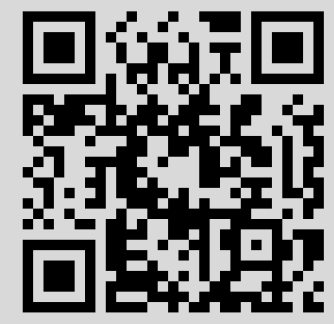


Используем эту систему уравнений и систему уравнений эволюции для $\psi$ при вычислении производной (14). Производную $\partial_{t} \varphi^{(2)}$ заменим в силу системы (15), производную $\partial_{t} \psi^{(2)}$ - в силу уравнений эволюции для $\psi^{(2)}$, производную $\partial_{t} U-$ в силу системы уравнений $(7)$. В результате в правой части формулы (14) не содержится выражений от производных по $t$. Далее, после преобразований интегралов эта формула приводится к виду (8).

\section{ЛитеРАТУРА}

1. Kaup D. J. J. Math. Anal. Appl., 54, No. 3, 849-864 (1976). 2. Герджиков B. C., Христов E. X. Матем. заметки, 28, № 4, 501-512 (1980). 3. Kaup D. J. SIAM J. Appl. Math., 31, No. 1, 121-133 (1976). 4. Кричевер И. М. УМН, 44, No. 2, 121184 (1989). 5. Fokas A. S., Ablowitz M. J. J. Math. Phys., 25, No. 8, 2494-2505 (1984). 6. Arcadiev V. A., Pogrebkov A. K., Polivanov M. C. Phys. D, 36, 189-197 (1989). 7. Кулии П. П., Липовский В. Д. Зап. научн. семин. ЛОМИ, 161, 54-71 (1987). 8. Villaroel J., Ablowitz M. J. Inverse Problems, 7, 451 (1991). 9. Sung L. Y., Fokas A. S. Preprint Institute for Nonlinear Studies, ins \#98, April, 1990.

Институт математики с ВЦ УНЦ РАН

Поступило в редакцию

7 февраля 1996 г.

В переработанном виде

9 января 1997 г.

УДК 513.83

\section{Почти евклидовы плоскости в $\ell_{p}^{n}$}

(C) 1998. Ю. И. ЛюБИЧ, О. А. ШАТАЛОВА

Под $\ell_{p}^{n}(p \geqslant 1)$ мы понимаем пространство $\mathbb{R}^{n}$, снабженное нормой

$$
\|x\|=\left(\sum_{k=1}^{n}\left|\xi_{k}\right|^{p}\right)^{1 / p}
$$

в канонических координатах. Вопрос о существовании евклидовых подпространств в $\ell_{p}^{n}$ естествен с геометрической точки зрения, а также тесно связан с различными задачами, возникающими в спектральной теории, численном анализе, теории чисел, теории представлений групп и т. д. (см. $[3,4,6,7])$. В настоящей заметке мы ограничимся рассмотрением двумерных подпространств (плоскостей).

Теорема 1 [3]. Если $\ell_{p}^{n}$ содержит евклидову плоскость, то $p \in 2 \mathbb{N}=$ $\{2,4,6, \ldots\}$.

Обратное утверждение верно при достаточно большом $n$.

Теорема $2[4,6]$. При $p \in 2 \mathbb{N}$ пространство $\ell_{p}^{n}$ содержит евклидову плоскость тогда и только тогда, когда $n \geqslant p / 2+1$.

При $n \leqslant p / 2$ естественно рассматривать « $\varepsilon$-евклидовы» плоскости с возможно меньшим значением $\varepsilon$. Конечно, при этом уже не нужны ограничения 
на $p$, а проблема оказывается связанной с классической теоремой Дворецкого [2], в силу которой для любого $\varepsilon>0$ сушествует $n(\varepsilon, p)$, такое, что при $n \geqslant n(\varepsilon, p)$ пространство $\ell_{p}^{n}$ содержит $\varepsilon$-евклидову плоскость. Иными словами, при $n \geqslant n(\varepsilon, p)$ сушествует линейное вложение $f: \ell_{2}^{2} \rightarrow \ell_{p}^{n}$, удовлетворяющее неравенству $\|f\| \cdot\left\|f^{-1}\right\| \leqslant 1+\varepsilon$, где $f^{-1}: \operatorname{Im} f \rightarrow \ell_{2}^{2}-$ левое обратное к $f$. Для наименьшего значения $n(\varepsilon, p)$ при $p \notin 2 \mathbb{N}$ имеется двусторонняя оценка

$$
a(p) \varepsilon^{-1 /(p+1)} \leqslant n(\varepsilon, p) \leqslant b(p) \varepsilon^{-1 /(p+1)} \ln ^{4} \varepsilon,
$$

являюшаяся частным случаем одной теоремы Бургейна и Линденштрауса [1]. (Явный вид коэффициентов $a(p)$ и $b(p)$ неизвестен.) Таким образом, если $p \notin 2 \mathbb{N}$, то $n(\varepsilon, p) \rightarrow \infty$ при $\varepsilon \rightarrow 0$ и фиксированном $p$, в то время как если $p \in 2 \mathbb{N}$, то $n(\varepsilon, p)=p / 2+1$, согласно теореме 2 , т. е. в этом случае $n(\varepsilon, p)$ не зависит от $\varepsilon$.

Предполагая, что $p \in 2 \mathbb{N}, 2 \leqslant n \leqslant p / 2$, мы рассмотрим вопрос о наименьшем $\varepsilon=\varepsilon_{n, p}$, при котором в $\ell_{p}^{n}$ имеется $\varepsilon$-евклидова плоскость. По соображениям компактности такое $\varepsilon_{n, p}$ существует. По теореме $2 \varepsilon_{n, p}=0$ при $n \geqslant p / 2+1$. Отметим, что одна из количественных форм теоремы Дворецкого (см. [5]) дает оценку $\varepsilon_{n, p} \leqslant c n^{-2}$, где $c$ - абсолютная константа. Таким образом, $\varepsilon_{n, p} \rightarrow 0$ при $n \rightarrow \infty$ равномерно по $p$. Ниже мы даем количественное уточнение этого факта.

Рассмотрим тригонометрический полином

$$
T_{n, p}(\varphi)=\left(\begin{array}{c}
p \\
p / 2
\end{array}\right)+2 \sum_{j=1}^{[p /(2 n)]}\left(\begin{array}{c}
p \\
p / 2-n j
\end{array}\right) \cos 2 n j \varphi
$$

Лемма. Имеет место оценка

$$
\varepsilon_{n, p} \leqslant\left(\frac{\max T_{n, p}(\varphi)}{\min T_{n, p}(\varphi)}\right)^{1 / p}-1 .
$$

ТЕОРема 3. Если $p=O(n)$, то $\varepsilon_{n, p}$ экспоненциально стремится $\kappa$ нулю.

В некотором диапазоне значений $(n, p)$ правая часть неравенства (1) может быть вычислена явно.

Teоpema 4. Ecлu $n>p / 4, m o$

$$
\varepsilon_{n, p} \leqslant\left(\frac{\left(\begin{array}{c}
p \\
p / 2
\end{array}\right)+2\left(\begin{array}{c}
p \\
p / 2-n
\end{array}\right)}{\left(\begin{array}{c}
p \\
p / 2
\end{array}\right)-2\left(\begin{array}{c}
p \\
p / 2-n
\end{array}\right)}\right)^{1 / p}-1 .
$$

Eсли $p / 8<n \leqslant p / 4$, mo

$$
\varepsilon_{n, p} \leqslant\left(\frac{\left(\begin{array}{c}
p \\
p / 2
\end{array}\right)+2\left(\begin{array}{c}
p \\
p / 2-n
\end{array}\right)+2\left(\begin{array}{c}
p \\
p / 2-2 n
\end{array}\right)}{\left(\begin{array}{c}
p \\
p / 2
\end{array}\right)-2\left(\begin{array}{c}
p \\
p / 2-n
\end{array}\right)+2\left(\begin{array}{c}
p \\
p / 2-2 n
\end{array}\right)}\right)^{1 / p}-1 .
$$


СлЕДСТВИЕ. Имеет место оченка

$$
\varepsilon_{p / 2, p} \leqslant\left(\frac{\left(\begin{array}{c}
p \\
p / 2
\end{array}\right)+2}{\left(\begin{array}{c}
p \\
p / 2
\end{array}\right)-2}\right)^{1 / p}-1 .
$$

Обозначим правую часть неравенства (2) через $\eta_{p}$. Очевидно, при $p \rightarrow \infty$

$$
\eta_{p} \sim \frac{4}{p}\left(\begin{array}{c}
p \\
p / 2
\end{array}\right)^{-1} .
$$

Оказывается, что оценка (2) завышена не более чем вдвое асимптотически.

ТеОрема 5. Имеет место неравенство

$$
\varepsilon_{p / 2, p} \geqslant \frac{2}{p}\left(\begin{array}{c}
p \\
p / 2
\end{array}\right)^{-1} .
$$

Представляется правдоподобным, что оценка (2) асимптотически точна.

$$
\text { ЛитеРАТУРА }
$$

1. Bourgain J., Lindenstrauss J. In: Springer Lect. Notes in Math., Vol. 1376, 1989, pp. 278-288. 2. Dvoretzky A. In: Proc. Symp. on Linear Spaces. Jerusalem, 1961, pp. 123-160. 3. Любич Ю. И. Сиб. матем. ж., 11, № 2, 358-369 (1970). 4. Lyubich Yu. I., Vaserstein L. N. Geom. Dedicata, 47, 327-362 (1993). 5. Milman V. D. In: Springer Lect. Notes in Math., 1317, 283-289 (1988). 6. Reznick B. Memoirs Am. Math. Soc., 96, 463 (1992). 7. Seidel J. J. Discrete Math., 136, 281-293 (1994).

Department of Mathematics

Поступило в редакцию Technion, Haifa, Israel 23 мая 1997 г.

УдК $517.984+517.5$

\title{
О спектрах малых колебаний струны при наличии вязкого трения на одном конце
}

\author{
(c) 1998. В. Н. ПивовАРчик
}

Задача, описывающая малые колебания струны, левый конец которой закреплен (свободен), а правый может двигаться с вязким трением перпендикулярно равновесной длине струны, имеет вид

$$
\begin{gathered}
\frac{d u^{\prime}}{d M(x)}+\lambda^{2} u=0, \\
u(\lambda, 0)=0 \quad\left(u_{-}^{\prime}(\lambda, 0)=0\right), \\
\lambda^{-1} u_{+}^{\prime}(\lambda, l)+i \nu u(\lambda, l)=0 .
\end{gathered}
$$

Смысл операции $d / d M(x)$ см. в [1], $M(x)$ - неотрицательная неубывающая функция распределения массы струны, $M(x+0)=M(x)$ для всех $x \in[0, l]$, и 